\title{
Geometric approach to non-scanned area for combined use of sector scanning sonar with quantitative echo-sounder
}

\author{
HIROSHI MAEDA, ${ }^{1}$ TAKESHI NAKAMURA ${ }^{1}$ AND AKIRA HAMANO ${ }^{1}$ \\ I National Fisheries University, 2-7-1, Nagata-honmachi, Shimonosehi, Yamaguchi 759-6595, Japan (ahamano@fish-u.ac.jp)
}

SUMMARY: Using the results from the coastal survey off Fukuoka Prefecture, the importance of using sonar for a clear understanding of the distribution of schools was shown by comparing the figure drawn based on the echo-sounder information with that based on the sector scanning sonar information. The stepwise transmission of a beam of a different tilt from a different position on the transect by sector scanning sonar caused the non-scanned volume. A geometric consideration revealed that the nonscanned volume was, under the present survey conditions, an upside-down triangle with a $32 \mathrm{~m}$ base and $65 \mathrm{~m}$ depth every $36 \mathrm{~m}$ on the section $20 \mathrm{~m}$ from the transect. Its apex first deepened then became shallower, and its base was shortened with an increase in the distance from the transect.

KEYWORDS: non-scanned volume, geometric approach, quantitative echo-sounder, sector scanning sonar, coastal echo survey

\section{INTRODUCTION}

Echo surveys could be classified into two types with respect of their scale, principal aim and the method of treating the obtained data. One is to access the standing stock of commercially important species which covers a very wide area as the base of setting the catch quota on a national or inter-regional level. ${ }^{1)}$ The obtained data were treated by application to the line transect theory. ${ }^{2}$ The other one is to estimate the distribution of available resources within a small area as the base for local development plan. The sounded data from this type of survey were treated in order to obtain detailed information about the distribution of specified or unspecified targets. The presence report deals with this type of survey results.

In the preceding report, ${ }^{3)}$ the additional use of scanning sonar with an echo-sounder was proposed for using the information in the area between the echo-sounded transects. However, there were many cases, in which a well-equipped vessel was unavailable. For these cases, a portable type sector scanning sonar is one of the most practical instruments. A sector scanning sonar stepwise transmits the sound with narrow beam of a different tilt sector by sector. A new problem, i.e., the presence of a non-scanned volume by the beams of the sector scanning sonar near the transects in the shallow layer, was introduced by this way of sonar beam transmission. The present report is to show the importance of the information from sonar, and to discuss geometrically the distance-dependence change in the form of non-scanned volume on the section parallel to the transects.
The data used in the present study were part of the results of the pelagic fish survey in December 1998, off Fukuoka Prefecture. In this survey, 22 parallel transects of 1 nautical mile long were settled at 0.1 nautical mile intervals in the direction nearly perpendicular to the coastal line. These transects were insonified from the vessel sailing at a speed of 3.5 knots using the quantitative echo-sounder of Simrad EY-500 and the sector scanning sonar of Furuno $\mathrm{CH}-34$. The settings of the respective parameters of these instruments for this survey are listed in Table 1.

The obtained data from these instruments were processed by the methods shown in the preceding report. ${ }^{4)}$

Table 1 Technical specification of echo-sounder and sector scaming somar

\begin{tabular}{|c|c|c|}
\hline & Echo-sounder & Sector scanning \\
\hline & Simrad EY-500 & Furuno $\mathrm{CH}-34$ \\
\hline Frequency $(\mathrm{kHz})$ & 70 & 162 \\
\hline \multicolumn{3}{|c|}{ Beam width (degree) } \\
\hline horizontal & - & 12 \\
\hline vertical & - & 9 \\
\hline $\begin{array}{l}\text { alongship } \\
\text { (=athwartship) }\end{array}$ & 11 & - \\
\hline Pulse length (ms) & $1.2^{*}$ & $0.33^{*}$ \\
\hline Ping rate (rate/s) & 1 & - \\
\hline $\begin{array}{r}\text { Time to complete } \\
\text { a sonar scan (s) }\end{array}$ & - & 10 \\
\hline
\end{tabular}

*Variable, depending on the values of the other factors

RESULTS AND DISCUSSION 
Horizontal distribution of schools detected by the quantitative echo-sounder

In the usual method of processing the echo survey data, the figure showing the horizontal distribution of the schools was drawn as if the information just underneath the transect was methodologically effective throughout the interval between the adjacent transects. The data obtained along the respective transects during the survey were processed by this usual method. ${ }^{2)}$ The detected distribution of schools is shown as Fig. 1, in which a value of $-55 \mathrm{~dB}$ was set as the threshold to remove the echoes from the boundaries and small floating objects such as plankton. This figure revealed that the target fish, mainly anchovy of Engraulis japonicus, were scattered into many small patches. Most of them were detected by the sounding not more than one transect. They covered short distance on the transects. This finding raised the question whether or not there remained unnegligible small patches, which were missed by the echo survey, because they happened to exist between the transects.

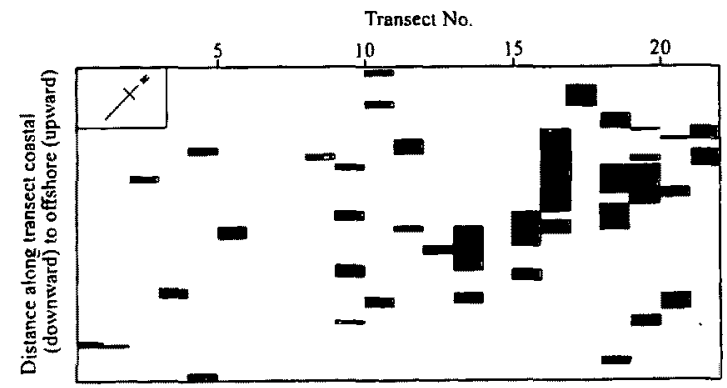

Fig.1 Projected distribution of patches on a horizontal plane, which were detected by the quantitative echo-sounder.

Horizontal distribution of schools detected by the sector scanning sonar

The three-dimensional image, which was drawn by the method shown in the preceding report, ${ }^{4)}$ was simplified and projected on a horizontal plane, and shown as Fig.2.

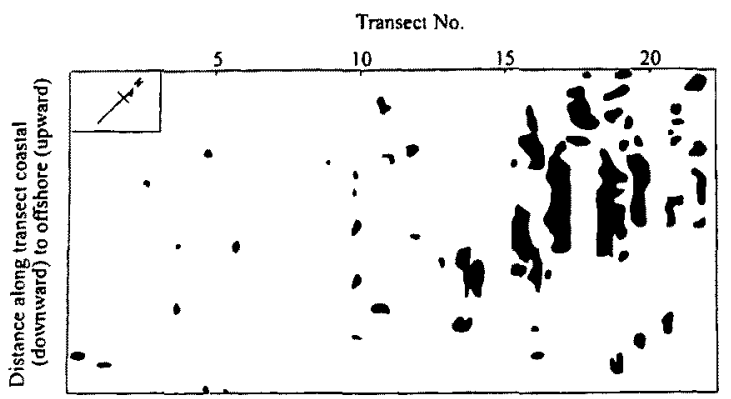

Fig.2 Projected distribution of patches on a horizontal plane, whicli were detected by the sector scanning sonar.
A comparison of Fig. 2 with Fig.1 showed that the patches, which were not detected by the echo sounding, were few. However, before concluding that there were few patches undetected by the echo sounding, it is necessary to give a geometric consideration on the non-scanned volume, which remained near the transect just underneath the surface due to the stepwise transmission of the sound beam of different tilt by the sector scanning sonar.

\section{Non-scanned area by the echo-sounder beam}

This equipment transmits the sound with narrow beam in a vertical direction at very short time intervals. The area covered by a ping of the sound beam touches that by the succeeding one at a point some distance apart from the sound source. The distance of this point from the line, which connects the start points of succeeding pings, is used in the present report as the shortest range of the fully searched distance and is abbreviated as SFD. SFD on this section is expressed $\left(d_{1} / 2\right) \cot (\alpha / 2)$, where $d_{1}$ is the distance sailed between the succeeding two pings and $\alpha$ is the angle of the echo-sounder beam. With the vessel sailing at 3.5 knots and the beam angle at its half angle, the SFD shape of the present case is an upside-down triangle of $9.3 \mathrm{~m}$ in height and $1.8 \mathrm{~m}$ in the base length. The possibility of a school undetected by the sounder beam exists only when a school is fully located within this triangle. When a layer shallower than $5 \mathrm{~m}$ was excluded because of surface noise, this possibility is negligibly small.

The possibility of a school, which was not detected, is large between the transects, because of the narrow horizontal coverage of the echo-sounder beam compared with the transect interval. The scarcity of a detected school in the left half and down half in Fig.1 is doubtful. This is the reason why the use of scanning sonar is proposed.

\section{Non-scanned volume by the sector scanning sonar beams}

Each ping differs in three factors - the tilt, location of the transmission on the transect, and swing direction. This sound beam transmission method makes it necessary to examine in detail the distribution of the non-scanned volume on the horizontal section and vertical one, ${ }^{5)}$ especially on the latter section. The stereographic image of the sector scanning sonar is given in Fig. 3.

Every two swings are needed to complete a cycle of scanning. Namely, the sector scamning sonar can collect the information in every other horizontal 
columns of $18 \mathrm{~m}$ width in the present case, changing the information from one side of the transect to that from the other side.

Horizontal section in the scanned half The shape of the non-scanned volume by the sonar beams on the horizontal section is similar to that by the sounder beams on the vertical section.

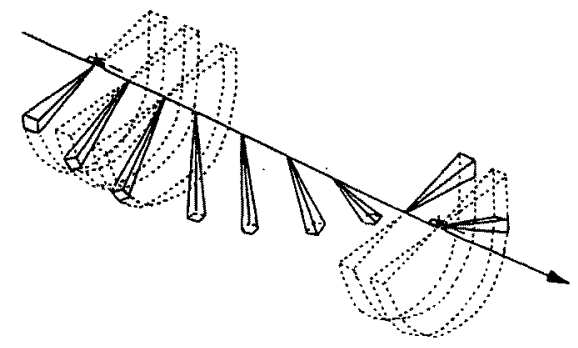

Fig. 3 Stereographic image of sector scanning sonar beanis.

Long pyramids are sonar beams. The intervals between the points of transmitting beam were elongated, because of showing clearly that the beams of different tilts were transmitted from the vessel sailing along the transect.

SFD is estimated to be $2.85 \mathrm{~m}$ by substituting $d_{2}$ for $d_{1}$ and $\beta$ for $\alpha$, where $d_{2}$ is the sailing distance between the succeeding two steps of the beam transmission by the sector scanning sonar and $\beta$ is the horizontal angle of the sonar beam. This is the shape of the non-scanned volume on the horizontal section, stacking the searched areas by the different tilts but neglecting the difference in depth. Further stereographic consideration is needed as the next step of the analysis.

Vertical section One of the key points of the present report, to which full attention in the examination for the sounded results should be paid, is the description of the non-scanned volume in the scanned half on this section. The section of sound beam along sound axis is circle or ellipse form. The beam with some tilt is projected on vertical section. However, to simplify the discussion, its projected image is set to be rectangle form. The sector scanning sonar initially transmits beam to horizontal direction, then does so increasing the tilt at 3 degree intervals step by step. The width and depth of the sound beam of the $i$-th step projected on the parallel section of $w_{1}$ apart from the transect is expressed as follows, where $w_{1}$ is the distance of a supposed vertical section from that along the transect:

The covered width by the beam of the $i$-th step $x_{1}$ to $x_{2}$ length of sound axis $b x=w_{1} \sec (i \delta)$

$x_{j}=i d_{2} \pm b x \tan (\beta / 2)$

- by one horizontal edge of sound beam $\left(x_{1}\right)$

+ by the other one

$\left(x_{2}\right)$ where $\delta$ is the pitch of the tilt of the sonar beam.

The covered depth range $z_{1}$ to $z_{2}$

$$
\begin{aligned}
z_{j}= & w_{1} \tan \{(i \pm 0.5) \gamma\} \\
& -\quad \text { by the shallowest end of beam }\left(z_{1}\right) \\
& +\quad \text { by the deepest one }\left(z_{2}\right)
\end{aligned}
$$

where $\gamma$ is the vertical angle of the sonar beam.

The shallower envelopes of the projected images of the respective sonar beams on a vertical section are shown in Fig.4. In this figure, non-scanned volume due to scanning to the other side of transect is also shown. With an increase in the tilt, the beam of the same angle covers wider area both horizontally and vertically. Also the resolution of the beam decreases with an increase in the tilt. Reasonable attention should be paid to this problem.

The projected images of the beam and the envelopes revealed the following fact. There remained the non-scanned area of the upside-down triangle-like form. This phenomenon occurs every $36 \mathrm{~m}$, including the non-scanned columns of $18 \mathrm{~m}$ width. The base is shortened and the apex is first deepened then became shallow with an increase in $w_{1}$.

The patch shown at the uppermost part of transect 10 (Fig.1) was detected by the echo-sounder but not by the sector scanning sonar (Fig.2). This patch was detected by the echo-sounder just underneath the surface, i.e. in the non-scanned volume by the sonar beam. This is the good example of showing the necessity of examining the location of non-scanned volume by the sonar beam.

Transverse section There is no problem with regard to the non-scanned volume when all the images on the vertical sections were stacked neglecting the difference in the transmitted position on the transect within a swing.

\section{Additional discussion}

The forms of the non-scanned volume by the sounder beams on the vertical and transverse sections were geometrically examined. There remained two additional problems - correction for the size of the school and for the low coverage by the sounder beams. A short description about the former problem of the echo-sounder was already given. A further tracing through the geometric approach is needed to connect the echo-sounder image with the location of the nonscanned volume ${ }^{6)}$ as pointed out by the comparison of Fig.1 with Fig.2.

The latter is the consideration of the extremely low coverage of the sounder beams in the transverse section, being $1.3 \%$. The rate of the scanned area depends on the depth and interval between the 
a) $w_{1}=20 \mathrm{~m}$

b) $w_{1}=45 \mathrm{~m}$

c) $w_{1}=90 \mathrm{~m}$

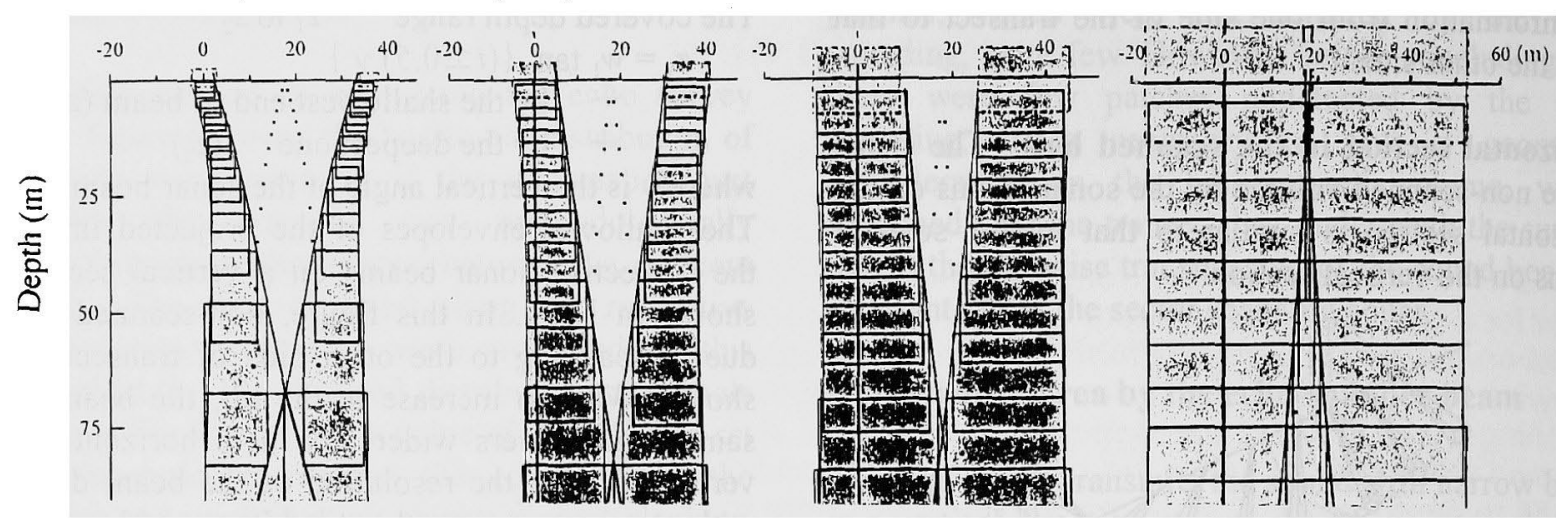

Fig. 4 Change in the form of the scanned area by sector scanning sonar beams on vertical section in accordance with distance from transect. Squares indicate the projected image of the respective sound beams. Lines show their envelopes and dots show the location of the axis of the respective sound beams swung to the other side of the transect. (Depth scale is slightly elongated)

transects. The scanned area increases in accordance with the increase in depth and the tangent of the beam angle $\alpha$. The influence of this problem is serious in the shallow water survey using a quantitative echo-sounder with narrow beam. In the present case, the information from the sonar is combined with that from the echosounder. However, the presence of the non-scanned volume by the sonar beam was pointed out. The probable way of escaping the influence of low coverage by the sounder beam may be found in the choice of the direction of the transects. One of the ways is to settle them in grid form.? Another way may be to settle them to cross the probable zonated distribution of targets. The fish distribute along the isobath or coastal line. This trend is clear in demersal fish, although no definite trend in this respect was found in pelagic fish. ${ }^{8)}$ The direction of the transects in the present case was chosen so as to perpendicularly cross the isobath.

The problem, which was newly caused by the combined use of the sector scanning sonar in the present report, and needed to be solved, is how to reduce the influence of the non-scanned volume on the vertical section by its beams. A helpful hint for this problem could be found in the following finding with regard to the non-scanned area on this section: The shallower envelope of one side of the sound beam in the deepening phase of the tilt in the figure, left side approaches that of the other side in the succeeding shallowing phase (in the figure, right side) in accordance with the increase in $w_{1}$. The nonscanned area disappears when $w_{1}$ exceeds some value, although the resolution of the image decreases with an increase in $w_{1}$ and the tilt. The distance of $160 \mathrm{~m}$ means a section $26 \mathrm{~m}$ apart from the preceding or succeeding transect. This finding suggested the effective use of the information from the scanning to the other side of the transect as shown in Fig.4 (d), for the purpose of eliminating the influence of the non-scanned area by sonar beams.

\section{REFERENCES}

1. Johannesson KA, Mitson RB. Fisheries Acoustics. A practical manual for aquatic biomass estimation. FAO Fisheries technical paper No.240. Roma, 1983,249 pp.

2. Simmonds EJ, Williamson NJ, Gerlotto F, Aglen A. Acoustic survey design and analysis procedure: A comprehensive review of current practice. ICES Cooperative research report No.187..Denmark, 1992, 127 pp.

3. Hamano A, Nakamura T. Combined use of quantitative echosounder with scanning sonar to visualize semi-quantitative three-dimensional image of school. J.NatFish. Univ. 2001; $50: 1-11$.

4. Hamano A, Nakamura T, Mizuguchi N. Quantitative. Assessment of small pelagic school based on a three-dimensional analysis using a scanning sonar and echo-sounder. The proceedings of the Intemational Symposium. Acoustgear 2000 (accepted).

5. Mitson RB. Fisheries Sonar. Fishing News Books Ltd, Farnham, Surrey, England, 1983, pp.194-232.

6. Gerlotto F, Soria M, Freon P. From two dimensions to threx: the use of multibeam sonar for a new approach in fisheries acoustics. Can.J.Fish Aquat. Sci. 1999; 56: 6-12.

7. Foote $\mathrm{KG}$, Stefansson $\mathrm{G}$. Definition of the problem of estimating fish abundance over an area from acoustic linetransect measurements of density. ICES JMar.Sci. 1993; 50:369-381.

8. Alveson DL. A study of amulal and seasonal bathymetric catch patterns for commercially important groundfishes of the Pacific Northwest Coast of North America. Pac. Mar. Fisheries Comm. Bull. 1960; 4: 1-60. 